\title{
Adiponectin suppresses gluconeogenic gene expression in mouse hepatocytes independent of LKB1-AMPK signaling
}

Russell A. Miller, ${ }^{1}$ Qingwei Chu, ${ }^{1}$ John Le Lay, ${ }^{1}$ Philipp E. Scherer, ${ }^{2}$ Rexford S. Ahima, ${ }^{1}$ Klaus H. Kaestner, ${ }^{1}$ Marc Foretz, ${ }^{3,4}$ Benoit Viollet, ${ }^{3,4}$ and Morris J. Birnbaum ${ }^{1}$

${ }^{1}$ The Institute of Diabetes, Obesity, and Metabolism, University of Pennsylvania, Philadelphia, Pennsylvania, USA.

${ }^{2}$ Touchstone Diabetes Center, The University of Texas Southwestern Medical Center, Dallas, Texas, USA.

${ }^{3}$ Institut Cochin, Université Paris Descartes, CNRS (UMR 8104), Paris, France. ${ }^{4}$ Inserm U1016, Paris, France.

\begin{abstract}
The adipocyte-derived hormone adiponectin signals from the fat storage depot to regulate metabolism in peripheral tissues. Inversely correlated with body fat levels, adiponectin reduction in obese individuals may play a causal role in the symptoms of metabolic syndrome. Adiponectin lowers serum glucose through suppression of hepatic glucose production, an effect attributed to activation of AMPK. Here, we investigated the signaling pathways that mediate the effects of adiponectin by studying mice with inducible hepatic deletion of LKB1, an upstream regulator of AMPK. We found that loss of LKB1 in the liver partially impaired the ability of adiponectin to lower serum glucose, though other actions of the hormone were preserved, including reduction of gluconeogenic gene expression and hepatic glucose production as assessed by euglycemic hyperinsulinemic clamp. Furthermore, in primary mouse hepatocytes, the absence of LKB1, AMPK, or the transcriptional coactivator CRTC2 did not prevent adiponectin from inhibiting glucose output or reducing gluconeogenic gene expression. These results reveal that whereas some of the hormone's actions in vivo may be LKB1 dependent, substantial LKB1-, AMPK-, and CRTC2-independent signaling pathways also mediate effects of adiponectin.
\end{abstract}

\section{Introduction}

The discovery of adipose tissue as an endocrine organ that can secrete adipokine hormones has provided new insights into the role of this tissue in the homeostasis of organismal nutrient metabolism. The identification and characterization of one such adipokine, adiponectin, revealed an intriguing interaction between adipocytes and nutrient metabolism in peripheral tissues $(1,2)$. The importance of adiponectin is heightened by the clear association of decreased adiponectin levels in obese individuals with an elevated risk for insulin resistance and cardiovascular disease $(3,4)$ and the identification of adiponectin missense mutations that associate with increased risk for insulin resistance and coronary artery disease $(5,6)$. While the precise mechanisms by which adiponectin may antagonize adverse outcomes are unclear, administration of adiponectin is known to increase fatty acid oxidation in metabolic tissues, decrease hepatic glucose production, increase glucose uptake in muscle cell culture, and alter food intake and energy expenditure through central actions $(5,7-11)$. For these reasons, adiponectin-mimetic therapies have become an attractive potential treatment for the metabolic syndrome.

Multiple studies have found that adiponectin lowers blood glucose acutely through a reduction in hepatic glucose output with little or no effect on glucose disposal $(9,10,12)$. Adiponectin also reduces hepatic and serum triglyceride levels and protects from both alcoholic and nonalcoholic hepatic steatosis $(7,13)$. Reduction of adiponectin in mice leads to impaired glucose tolerance and elevated hepatic glucose production, further corroborating the role for adiponectin in normal hepatic metabolism (14). The role has been further established in studies showing that the reversal of

Conflict of interest: The authors have declared that no conflict of interest exists. Citation for this article: J Clin Invest. 2011;121(6):2518-2528. doi:10.1172/JCI45942. decreased serum adiponectin levels in $o b / o b$ mice through transgenic expression can dramatically improve their metabolic profile (15). The glucose-lowering functions of adiponectin have been attributed to the hepatic activation of AMPK, providing a mechanistic link to a signal transduction pathway already established as an antagonist of hepatic glucose output and lipogenesis (16).

AMPK is an evolutionarily conserved protein kinase that serves as a primary cellular monitor of energy charge; conditions that deplete energy and thus elevate the AMP/ATP ratio activate the kinase and result in a decrease in anabolic and increase in catabolic pathways (17). AMPK is a heterotrimeric enzyme, consisting of regulatory $\beta$ and $\gamma$ subunits and a catalytic $\alpha$ subunit that requires phosphorylation by an upstream AMPK kinase (AMPKK) to achieve full activity. Once activated, AMPK phosphorylates target proteins such as acetyl-CoA carboxylase (ACC) and CREB-regulated transcription coactivator (CRTC2), also known as transducer of regulated cAMP response element-binding protein 2 (TORC2), restoring the energy status through multiple mechanisms including increased fatty acid oxidation and glucose uptake in muscle, and decreased hepatic lipogenesis and gluconeogenesis (16-19). The principle AMPKKs are the kinases STK11/LKB1 and CaMKK $\beta$, which phosphorylate AMPK in its activation loop at threonine 172 (20-23). In many cells, LKB1 is the dominant AMPKK, as its loss decreases basal and stimulated AMPK phosphorylation and activity (24-26). For example, deletion of LKB1 in liver elevates serum glucose levels and impairs glucose tolerance, which is associated with a significant loss of basal and metformin-stimulated AMPK phosphorylation (26). These data establish LKB1 as an important regulator of hepatic glucose production.

AMPK mediates some hepatic actions of adiponectin as evidenced by loss of adiponectin-induced glucose lowering in mice expressing a dominant negative AMPK protein in liver (27). More- 
A

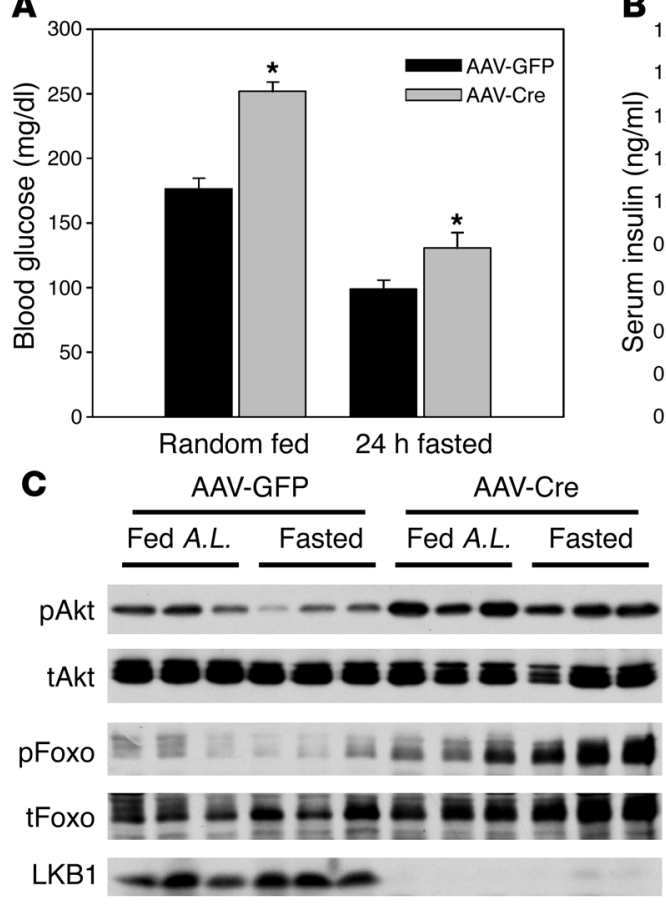

B

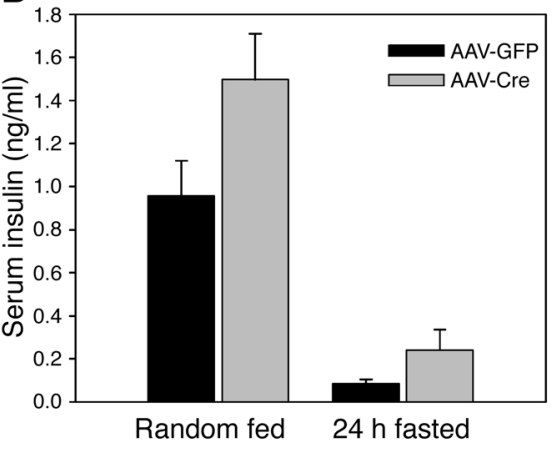

D

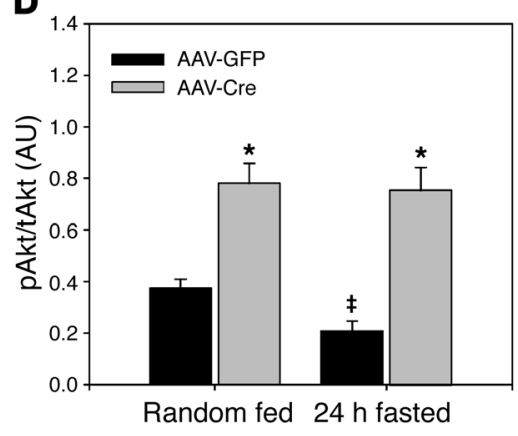

E

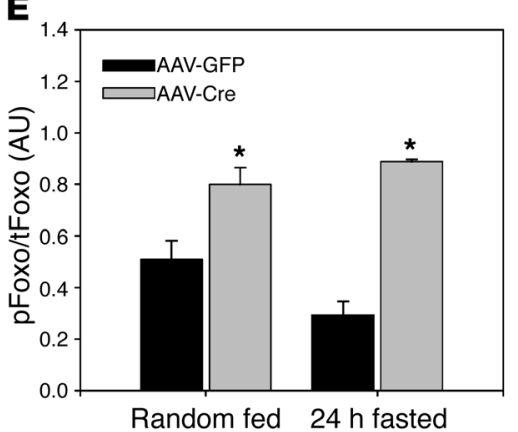

H

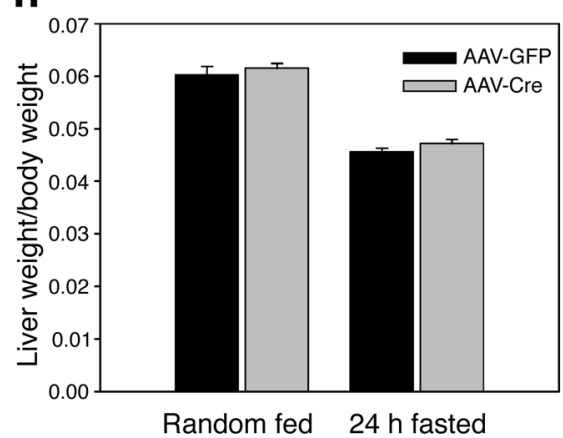

Random fed $24 \mathrm{~h}$ fasted

Random fed $24 \mathrm{~h}$ fasted
F

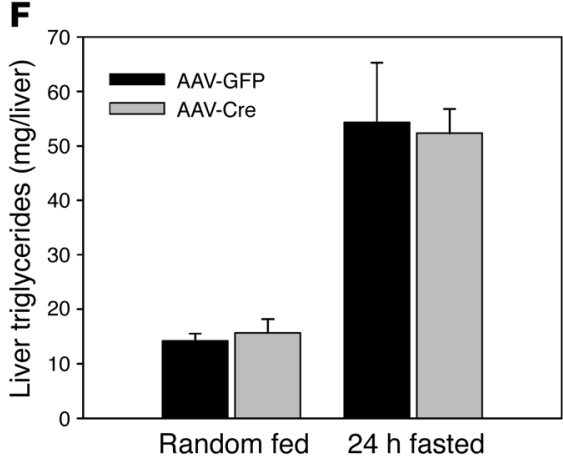

G

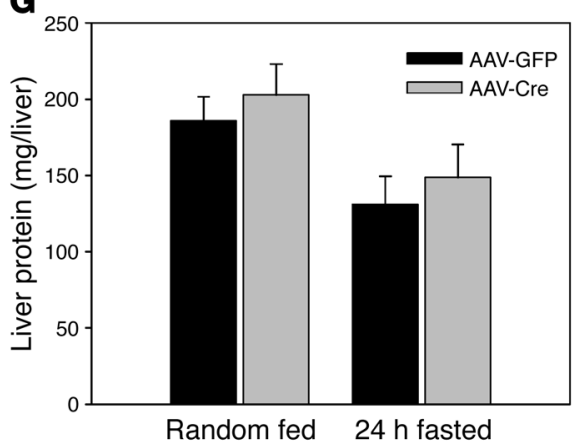

Figure 1

Loss of hepatic LKB1 leads to elevated serum glucose in fasted and fed-ad libitum mice. LKB1/ox/lox mice were infected with adeno-associated virus expressing either GFP or Cre recombinase 16 days prior to study. Mice were either fasted for 18 hours prior to sacrifice or fed ad libitum and sacrificed at $10 \mathrm{am}$. We quantified (A) blood glucose values and (B) serum insulin values from mice under the indicated conditions. (C) Liver tissue was extracted and Western blotted for phosphorylated Akt (T473), total Akt, phosphorylated Foxo1 (T26), total Foxo1, and total LKB1 protein. A.L., ad libitum. (D and E) Western blots were quantified and the phosphorylated/total Akt and Foxo ratio is shown. Total hepatic $(\mathbf{F})$ triglycerides and $(\mathbf{G})$ protein were quantified and $(\mathbf{H})$ liver weight/body weight ratio calculated. ${ }^{*} P<0.05$, GFP versus Cre; ${ }^{\ddagger} P<0.05$, fasting versus fed. All results are expressed as the mean, and error bars represent SEM.

over, AMPK and its activating kinase LKB1 are required for adiponectin to reduce the expression of the lipogenic gene Srebf1c (28). While these studies suggest that some effects of adiponectin depend on AMPK, it is unclear whether this pathway accounts for all the actions of this adipokine. Indeed, recent studies employing genetic loss of LKB1 and AMPK activities have revealed that the biguanide metformin does not require these proteins for its effects on glucose output or gluconeogenesis (29). Moreover, the precise contribution of LKB1, perhaps as a regulator of AMPKrelated kinases, to adiponectin signaling remains unclear. For these reasons, we utilized a genetic loss-of-function approach to ask whether LKB1 is required in vivo for the hepatic functions of adiponectin. We have taken advantage of a floxed LKB1 allele to conditionally delete the LKB1 protein in the liver, the major tissue responsible for the glucose-lowering effects of adiponectin (10). We have found that, while a component of adiponectin's lowering of hepatic glucose production is LKB1 dependent, the hormone still reduces hepatic glucose production and gluconeogenic mRNA expression in vivo in the absence of LKB1.

We have also utilized primary mouse hepatocytes in vitro to further characterize adiponectin-dependent control of glucose metabolism. One postulated mechanism for LKB1 and AMPK modulation of gluconeogenic mRNAs is through antagonism of the cAMP-responsive CREB signaling system at the level of the coactivator CRTC2. AMPK and other LKB1-dependent kinases phosphorylate and inactivate CRTC2, leading to reductions in the transcription of the Ppargc1a, Pck1, and G6pc genes (19). We therefore tested to determine whether the LKB/AMPK/CRTC2 signaling 
A

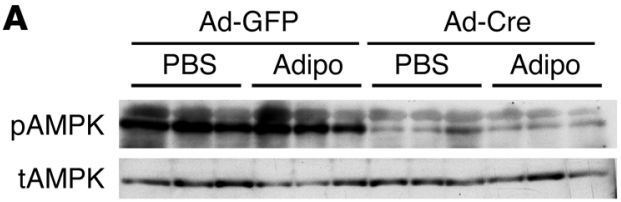

LKB

B

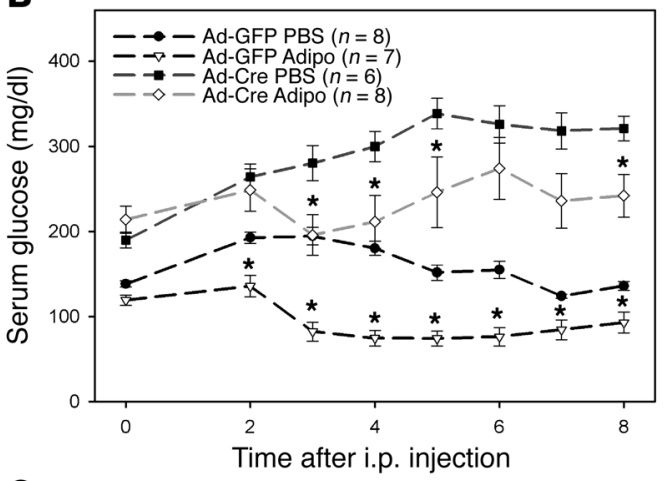

C

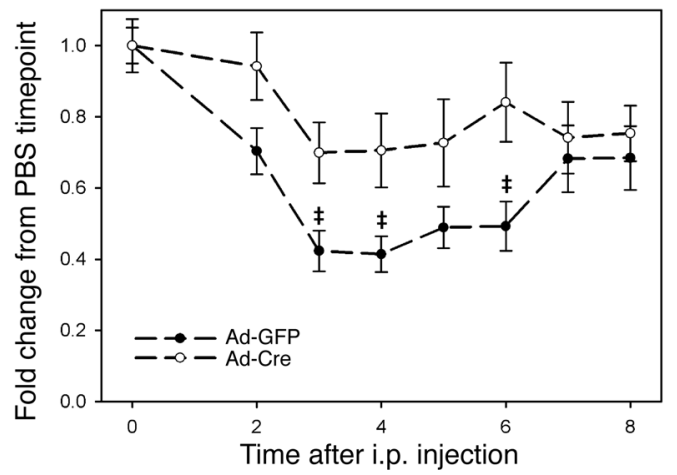

pathway was required for adiponectin-controlled gluconeogenic mRNA expression and glucose production in primary hepatocytes. Using cells isolated from LKB1-, AMPK $\alpha$-, or CRTC2-null livers, we found that the LKB1/AMPK/CRTC2 signaling axis is not necessary for regulation of glucose output or transcript levels of gluconeogenic enzymes in response to adiponectin, suggesting that adiponectin regulates hepatic metabolism by an LKB1-, AMPK-, and CRTC2-independent pathway.

\section{Results}

Conditional deletion of hepatic LKB1 results in elevated blood glucose levels. To evaluate the role of hepatic LKB1 in adiponectin action, we deleted acutely the STK11/LKB1 gene in the liver by viral delivery of the Cre recombinase to adult mice that were homozygous for a floxed allele of $L K B 1$ (30) and evaluated their metabolic phenotype. Glucose tolerance tests performed at varied intervals after viral delivery of Cre recombinase revealed a sustained glucose intolerance that was evident 11 days after infection (Supplemental Figure 1; supplemental material available online with this article; doi:10.1172/JCI45942DS1), as previously reported (26); loss of hepatic LKB1 in AAV-Cre-treated mice led to elevated serum glucose levels under both fasted and fed conditions (Figure 1A). Consistent with elevated blood glucose levels, serum insulin values trended to be increased in the absence of hepatic LKB1 and

\section{Figure 2}

Hepatic loss of LKB1 leads to reduced efficacy of adiponectin. $L K B 1$ lox/lox mice were infected with adenovirus expressing either GFP or Cre recombinase 16 days prior to study. (A) Western blots of liver tissue 8 hours after injection with either PBS or $34 \mu \mathrm{g} / \mathrm{g}$ body weight adiponectin (adipo) showing excision of LKB1 protein and concomitant reduction in AMPK T172 phosphorylation. (B) Blood glucose levels were obtained from infected mice injected i.p. with either PBS or $34 \mu \mathrm{g} / \mathrm{g}$ body weight adiponectin. (C) Fold change in blood glucose levels of adiponectin versus PBS injected at the indicated time point. ${ }^{*} P<0.05$ versus $P B S$ treatment of same genotype at equivalent time points; $\ddagger P<0.05$ GFP versus Cre. All results are expressed as the mean, and error bars represent SEM.

there was an elevation in hepatic Akt and Foxo1 phosphorylation under both fasted and fed conditions (Figure 1, B-E). Loss of hepatic LKB1 did not lead to alteration in liver triglycerides, protein, or the liver-to-body weight ratio under these conditions compared with AAV-GFP-treated control mice (Figure 1, F-H), suggesting that after removal of LKB1, the primary hepatic phenotype is altered glucose homeostasis.

Conditional deletion of hepatic LKB1 partially abrogates the glucose-lowering effects of adiponectin. To assess the requirement for LKB1 in adiponectin action, we injected adiponectin i.p. and monitored the blood glucose levels of these mice for 8 hours. We were unable to detect phosphorylation of AMPK in response to this adiponectin treatment in either the presence or absence of LKB1 (Figure 2A). Nonetheless, this dose of adiponectin was active, as Ad-GFPinfected $L K B 1^{l o x} /$ lox mice responded to adiponectin treatment with a significant reduction in serum glucose levels when compared with PBS-injected control mice, which displayed an increase from baseline, presumably due to the stress of the injection (Figure 2B). Serum glucose levels in adiponectin-treated, Ad-Cre-infected $L K B 1^{10 x / 10 x}$ mice were only partially lowered (Figure $2 B$ ). When the data from the adiponectin-treated mice were normalized to the PBS treatment group from corresponding genotypes, the dependence on LKB1 became more obvious (Figure 2C). These data largely replicate the adiponectin resistance produced by expression of a dominant inhibitory mutant of AMPK in liver (27).

LKB1 excision in liver partially blocks the effects of adiponectin on hepatic glucose production. We were concerned that the elevated glucose levels of hepatic LKB1-deficient mice at the beginning of the adiponectin treatment might have confounded interpretation of these experiments, as hyperglycemia could lead to effects unrelated to deletion of $L K B 1$. We therefore sought to control for these high glucose levels by comparing the effects of adiponectin in Ad-Creand Ad-GFP-infected LKB1 $1^{\text {lox } / \text { lox }}$ mice that were "clamped" at euglycemia by infusion of insulin and glucose. This experiment also allowed the direct measurement of hepatic glucose output. Mice infected with Ad-Cre again showed a dramatic loss of LKB1 protein (Figure 3A). Consistent with previous results in wild-type mice, intravenous infusion of adiponectin into Ad-GFP-infected $L K B 1^{10 x / l o x}$ mice for the 2-hour duration of the clamp procedure resulted in an increased glucose infusion rate, which was mediated by a reduction in hepatic glucose production with no significant change in the rate of glucose disposal (ref. 10; Figure 3, B, C, E, and F). Ad-Cre-infected LKB1-deficient mice exhibited a slightly lower glucose infusion rate and a statistically significant elevation of hepatic glucose production and no change in the rate of glucose disposal or tissue uptake, establishing a role for hepatic LKB1 in 
A
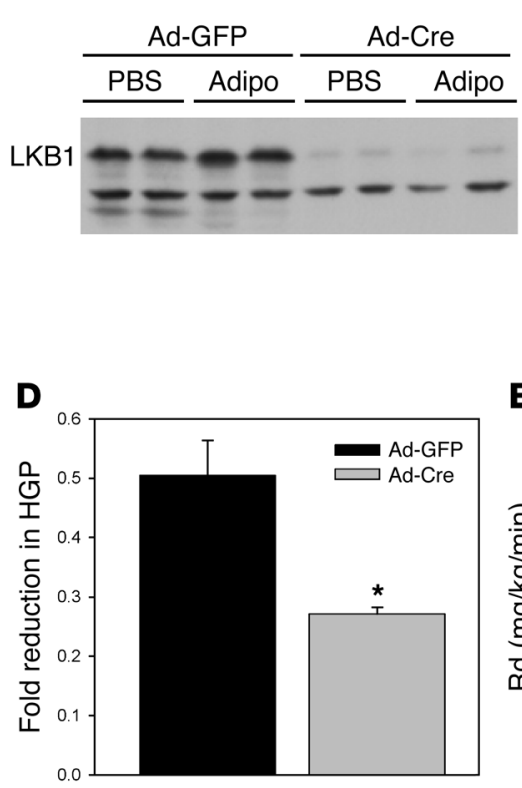

B

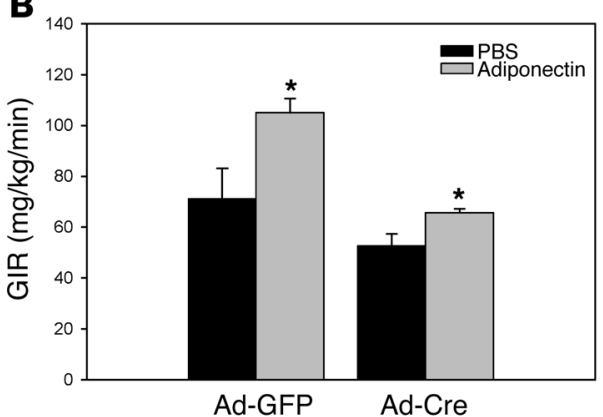

$\mathbf{E}$

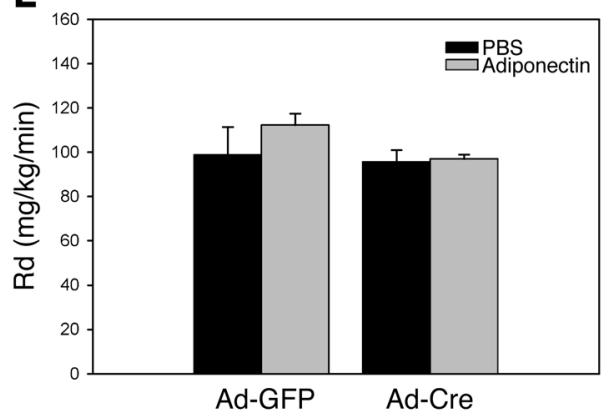

C

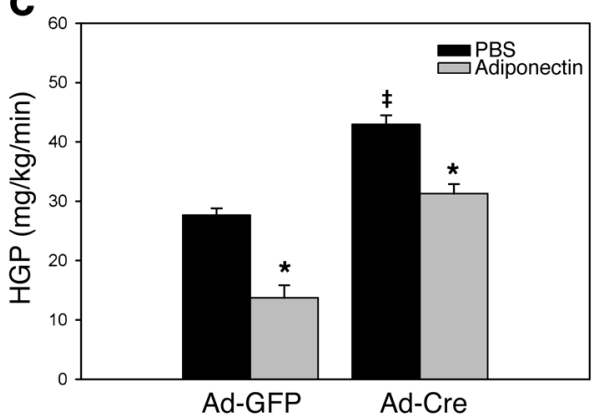

$\mathbf{F}$

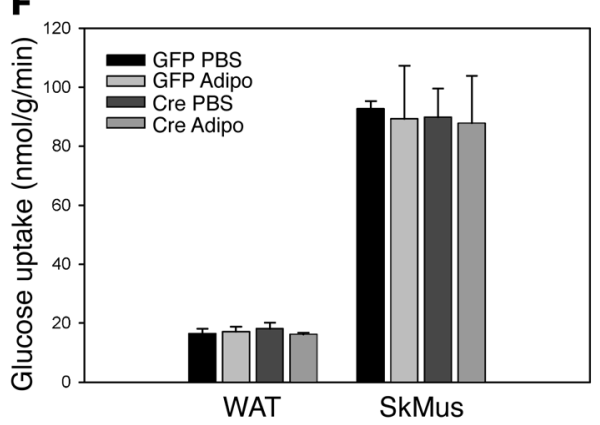

Figure 3

Hepatic loss of LKB1 alters adiponectin's effects on hepatic glucose production. Hyperinsulinemic, euglycemic clamp studies of adenovirusinfected $L K B 1^{10 x / l o x}$ mice infused i.v. with PBS or $50 \mathrm{ng} / \mathrm{min} / \mathrm{g}$ body weight of adiponectin were performed as indicated in Methods. Each group consisted of 4 mice. (A) Western blots from liver collected from mice following clamp experiments exhibiting loss of LKB1 protein. (B) Glucose infusion rate (GIR) and (C) hepatic glucose production (HGP) rate determined from clamped animals. (D) The fold reduction in hepatic glucose production due to adiponectin infusion was calculated for both groups of mice. (E) The rate of glucose disposal $(\mathrm{Rd})$ and $(\mathbf{F})$ rate of glucose uptake into white adipose tissue (WAT) and skeletal muscle (SkMus) for each group. ${ }^{*} P<0.05$, PBS versus adiponectin; ${ }^{\ddagger} P<0.05$, GFP versus Cre. All results are expressed as the mean, and error bars represent SEM.

basal glucose metabolism (Figure 3, B, C, E, and F). Surprisingly, adiponectin infusion in the LKB1-deficient mice still suppressed hepatic glucose production; however, this reduction was less than that produced by adiponectin in control animals (50\% vs. $27 \%$ reduction $[P<0.01]$ in Ad-GFP vs. Ad-Cre animals, respectively), suggesting that there are both LKB1-dependent and -independent components of adiponectin-induced reduction of hepatic glucose production (Figure 3, C and D). However, as noted above, the major impact of hepatic LKB1 deletion was to increase basal glucose output in the absence of exogenous adiponectin (Figure $3 \mathrm{C}$ ).

Loss of LKB1 affects basal gene expression but not the effects of adiponectin. To elucidate the mechanism by which LKB1 mediates the adiponectin-dependent reduction in hepatic glucose production, we studied the effect of i.p. injection of adiponectin on gene expression of a group of genes encoding proteins relevant to metabolic phenotypes of the liver. In control, Ad-GFP-infected $L K B 1^{l o x / l o x}$ animals, treatment with adiponectin reduced the mRNA of gluconeogenic and lipogenic genes Ppargc1a, G6pc, Pck1, Srebf1c, Acaca, Acly, and Fasn, consistent with the effect of the hormone in suppressing the anabolic program (Figure 4). In Ad-Cre-infected $L K B 1^{l o x} /$ lox animals, the loss of hepatic LKB1 increased the basal expression of many of the genes, including Ppargc1a, G6pc, Srebf1c, Acaca, Acly, and Fasn (Figure 4). These data emphasize that the degree of recombination and consequent reduction in LKB1 is sufficient to have biological consequences in vivo. Treatment of these mice with adiponectin markedly reduced the transcripts encoding these anabolic genes, but surprisingly, excision of LKB1 by infection with Ad-Cre was largely without effect on the action of adiponectin (Figure 4). These data indicate that LKB1 is required for maintenance of basal gene expression but is dispensable for signaling by adiponectin to the nucleus. Again, to eliminate the potential confounding effects of hyperglycemia, mRNA encoding gluconeogenic proteins was measured in livers at the termination of the euglycemic, hyperinsulinemic clamps. Under these conditions, loss of LKB1 resulted in significantly increased levels of Ppargc1a, Pck1, and G6pc mRNA, which were nonetheless reduced by adiponectin treatment independent of the presence of hepatic LKB1 (Supplemental Figure 2, B-D).

Activation of $A M P K$ by adiponectin is rapid, modest, and accompanied by elevated AMP levels. Prior work has suggested that adiponectin exerts its effects on AMPK through an increase in intracellular AMP, though this remains controversial $(27,31)$. To examine this question in primary hepatocytes, a physiological target tissue for adiponectin, we quantified the intracellular adenine nucleotide levels following treatment with adiponectin and other known AMPK activators. Traditional AMPK activators led to a robust increase in AMPK phosphorylation at T172 in primary hepatocytes in a time-dependent manner that was accompanied by a concomitant increase in cellular AMP levels, and at latter time points alterations in ADP and ATP levels (excluding AICAR [5-Aminoimidazole-4carboxamide ribonucleotide] and its monophosphorylated species ZMP) (Supplemental Figure 3). In contrast, adiponectin treatment elicited a modest and transient activation of AMPK, which peaked after 5 minutes, and was also correlated with a transient elevation 

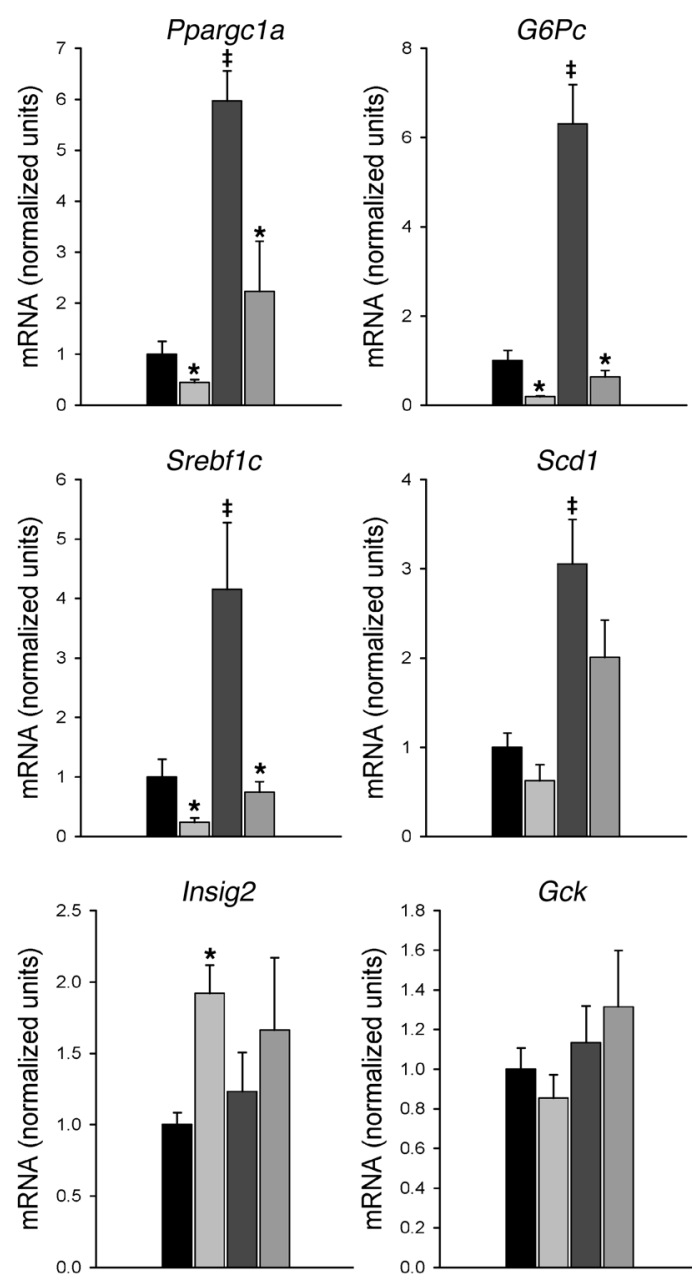
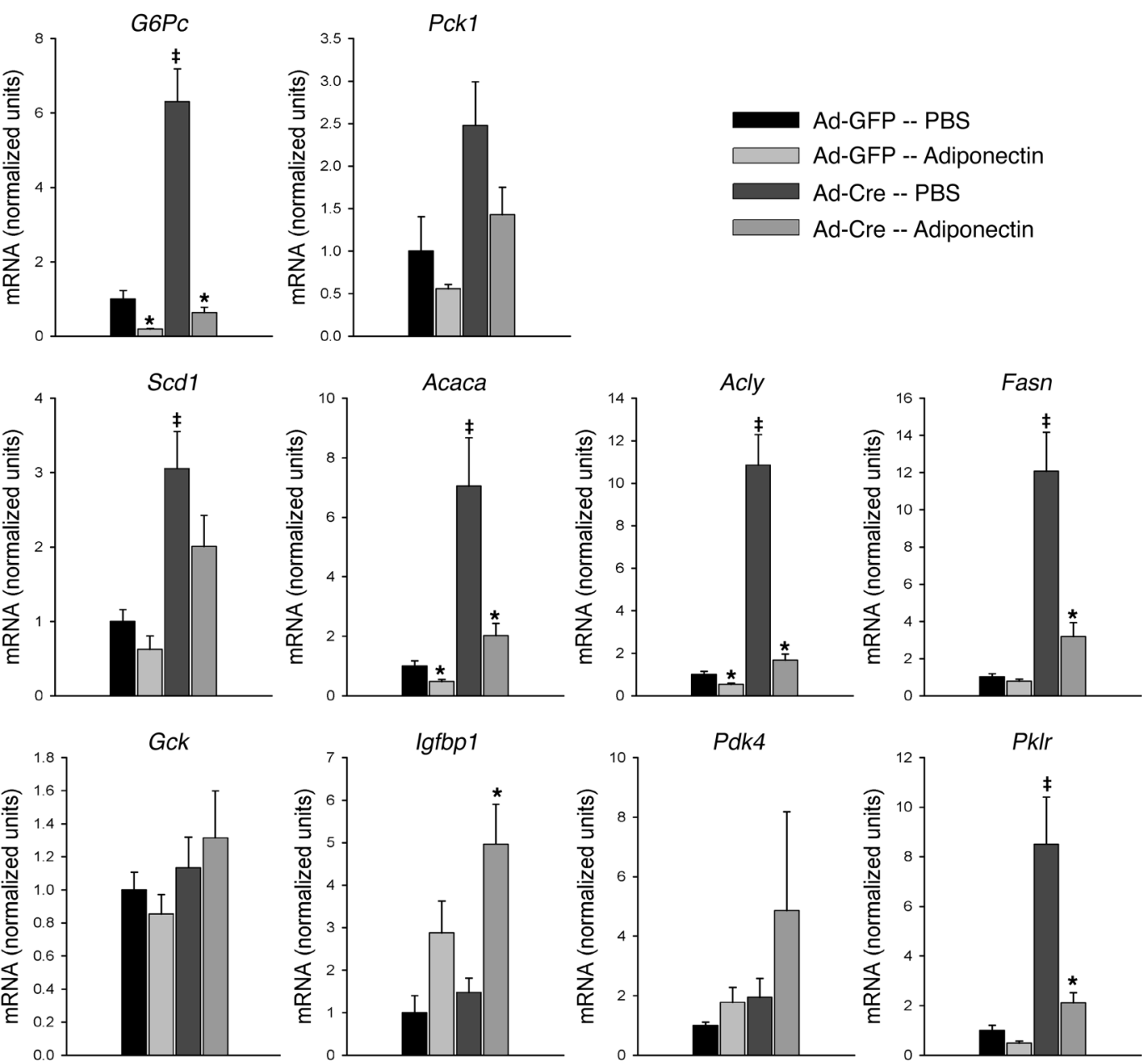

Figure 4

Adiponectin-induced gene expression following adiponectin tolerance test is not LKB1 dependent. Adenovirus-infected LKB1/ox/lox mice were injected i.p. with PBS or $34 \mu \mathrm{g} / \mathrm{g}$ body weight adiponectin, and 6 hours later, their livers were collected for mRNA analysis. The indicated mRNAs were quantified and expressed relative to Tbp mRNA levels. The data are represented normalized to the GFP-infected, PBS-injected group. ${ }^{*} P<0.05$ PBS versus adiponectin; ${ }^{\ddagger} P<0.05$ GFP versus Cre. All results are expressed as the mean, and error bars represent SEM.

in cellular AMP levels. We did not observe any alterations in phosphorylation of LKB1 after treatment with these AMPK activators (Supplemental Figure 3A).

$L K B 1$ is required for adiponectin-dependent activation of AMPK in primary bepatocytes. The effect of adiponectin on lowering hepatic glucose production could be exerted through adiponectin receptors on hepatocytes or nonautonomously via pathways involving communication from other organs. To address this question, we isolated primary hepatocytes from $L K B 1^{l o x} / l o x$ mice injected with Ad-GFP or Ad-Cre to assess the direct effects of adiponectin in vitro. AICAR, phenformin, and adiponectin all promoted phosphorylation of AMPK to varying degrees (Figure 5A). Injection of Ad-Cre 21 days prior to isolation of hepatocytes led to a substantial reduction in LKB1 as well as complete abrogation of adiponectin-, AICAR-, and phenformin-stimulated phosphorylation of AMPK and its target protein Raptor (Figure 5A). A modest phosphorylation of the AMPK substrate ACC persisted in LKB1-deficient cells, likely due to the presence of a small number of uninfected hepatocytes. These results indicate that LKB1 is the predominant upstream AMPKK in primary hepatocytes in response to these agonists in vitro and is required for adiponectin-dependent phosphorylation of AMPK. Moreover, these data again highlight the transient and modest activation of AMPK following adiponectin treatment when compared with other pharmacological AMPK activators.

LKB1 is not required for adiponectin-modulated gene expression and glucose production in primary hepatocytes. As presented above, loss of liver LKB1 had minimal effect on adiponectin-induced changes in hepatic gene expression in vivo. To determine whether this function of adiponectin represented a cell-autonomous effect of the hormone independent of hepatic LKB1, we evaluated the consequences of treating isolated hepatocytes with adiponectin. By 6 hours after addition of adiponectin, the transient increase in AMPK phosphorylation was ended, in contrast to the sizeable response to AICAR or phenformin (Figure 5B). In hepatocytes from control Ad-GFP-infected $L K B 1^{l o x} /$ lox animals, treatment with the nonhydrolyzable cyclic AMP analogue dibutyryl-cAMP (db-cAMP) increased markedly the accumulation of G6pc, Ppargc1a, and Pck1 mRNA, and this was antagonized by adiponectin or AICAR, with the latter being a more potent inhibitor (Figure 5, C-E). LKB1-deleted hepatocytes exhibited elevated basal mRNA levels for Pck1, G6pc, and Ppargc1a, and db-cAMP further increased the mRNA 
A

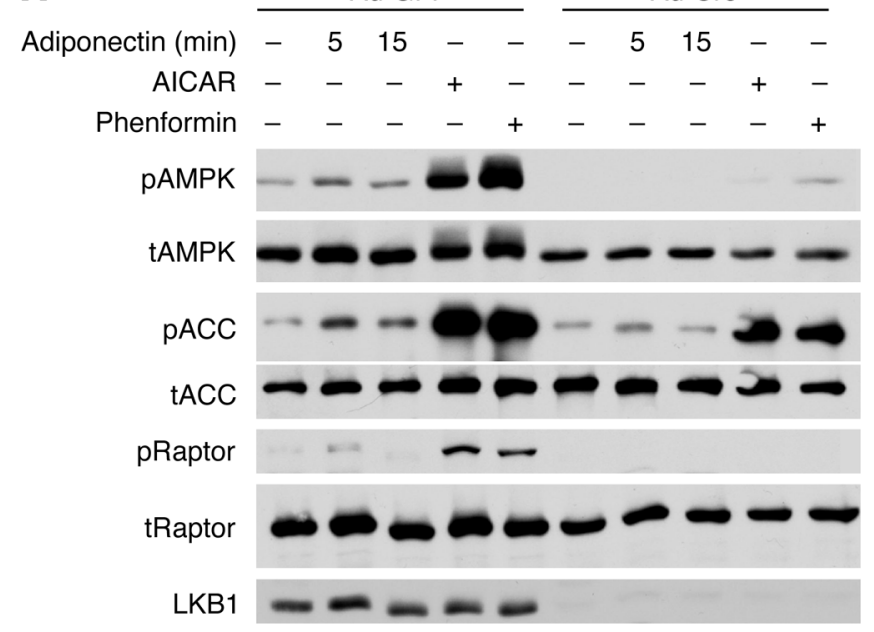

C

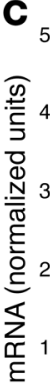

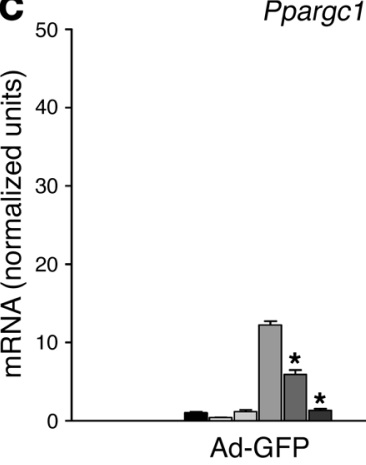

E

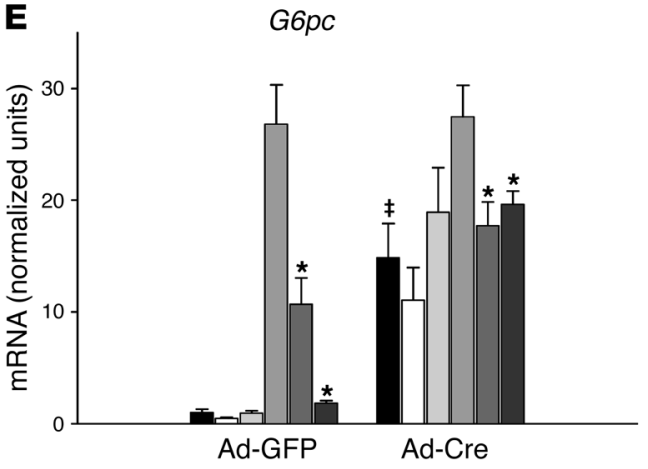

B

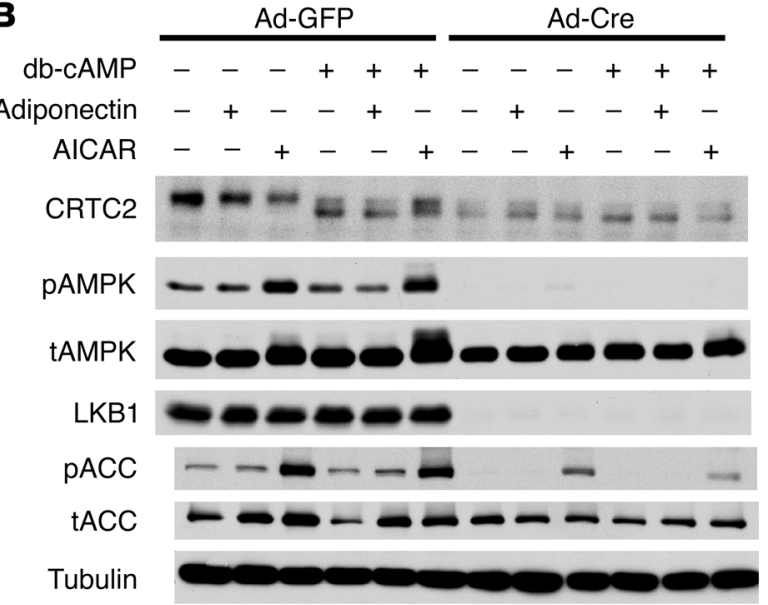

D

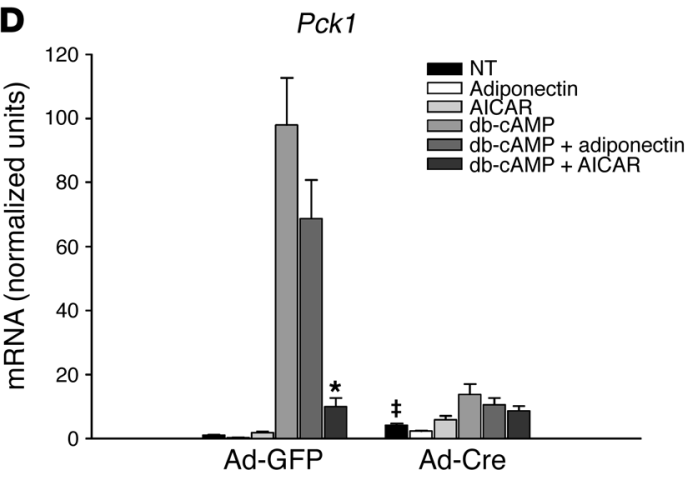

F

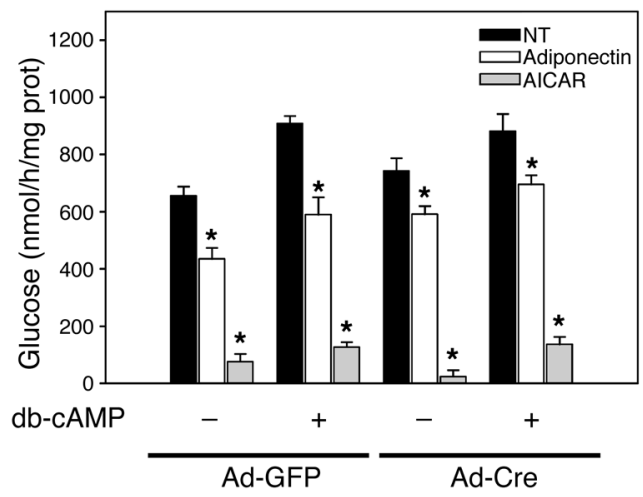

Figure 5

Adiponectin signaling in LKB1-deficient primary hepatocytes. (A) Primary hepatocytes isolated from adenovirus-infected $L K B 1$ lox/lox mice were treated with $30 \mu \mathrm{g} / \mathrm{ml}$ adiponectin (Adipo) for 5 or 15 minutes, $1 \mathrm{mM}$ AICAR for 1 hour, or $1 \mathrm{mM}$ phenformin (Phen) for 1 hour, and Western blots examining AMPK phosphorylation (pAMPK), total and phosphorylated ACC (tACC and pACC), total and phosphorylated Raptor (tRaptor and pRaptor), and LKB1 proteins were performed. (B) Western blots from hepatocytes isolated from adenovirus-infected $L K B 1$ lox/lox mice were treated with $30 \mu \mathrm{g} / \mathrm{ml}$ adiponectin, $500 \mu \mathrm{M}$ AICAR, and $100 \mu \mathrm{M}$ db-cAMP for 6 hours and probed for CRTC2, total AMPK (tAMPK) and phosphorylated AMPK, LKB1, total and phosphorylated ACC, and tubulin. (C-E) Primary hepatocytes isolated from adenovirus-infected LKB1 10x/lox mice were treated with $30 \mu \mathrm{g} / \mathrm{ml}$ adiponectin, $500 \mu \mathrm{M}$ AICAR, and $100 \mu \mathrm{M} \mathrm{db}$-cAMP for 6 hours. Total RNA was isolated and (C) Ppargc1a, (D) Pck1, and (E) G6pc mRNA was quantified and expressed relative to cyclophilin A mRNA and normalized to basal mRNA from GFP-infected hepatocytes. ${ }^{*} P<0.05$ vs. db-cAMP; $¥ P<0.05$ GFP versus Cre. (F) Primary hepatocytes isolated from adenovirus-infected $L K B 1^{10 x / l o x}$ mice were treated with $30 \mu \mathrm{g} / \mathrm{ml}$ adiponectin, $500 \mu \mathrm{M}$ AICAR, and $100 \mu \mathrm{M}$ db-cAMP for 6 hours in glucose-free medium, and glucose production was measured and normalized to total protein. ${ }^{*} P<0.05$ vs. untreated with adiponectin or AICAR. All results are expressed as the mean, and error bars represent SEM. 
A

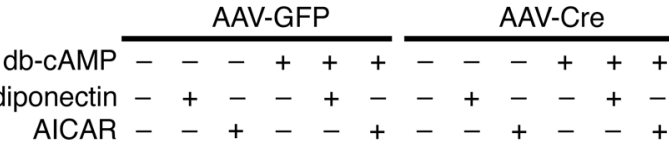
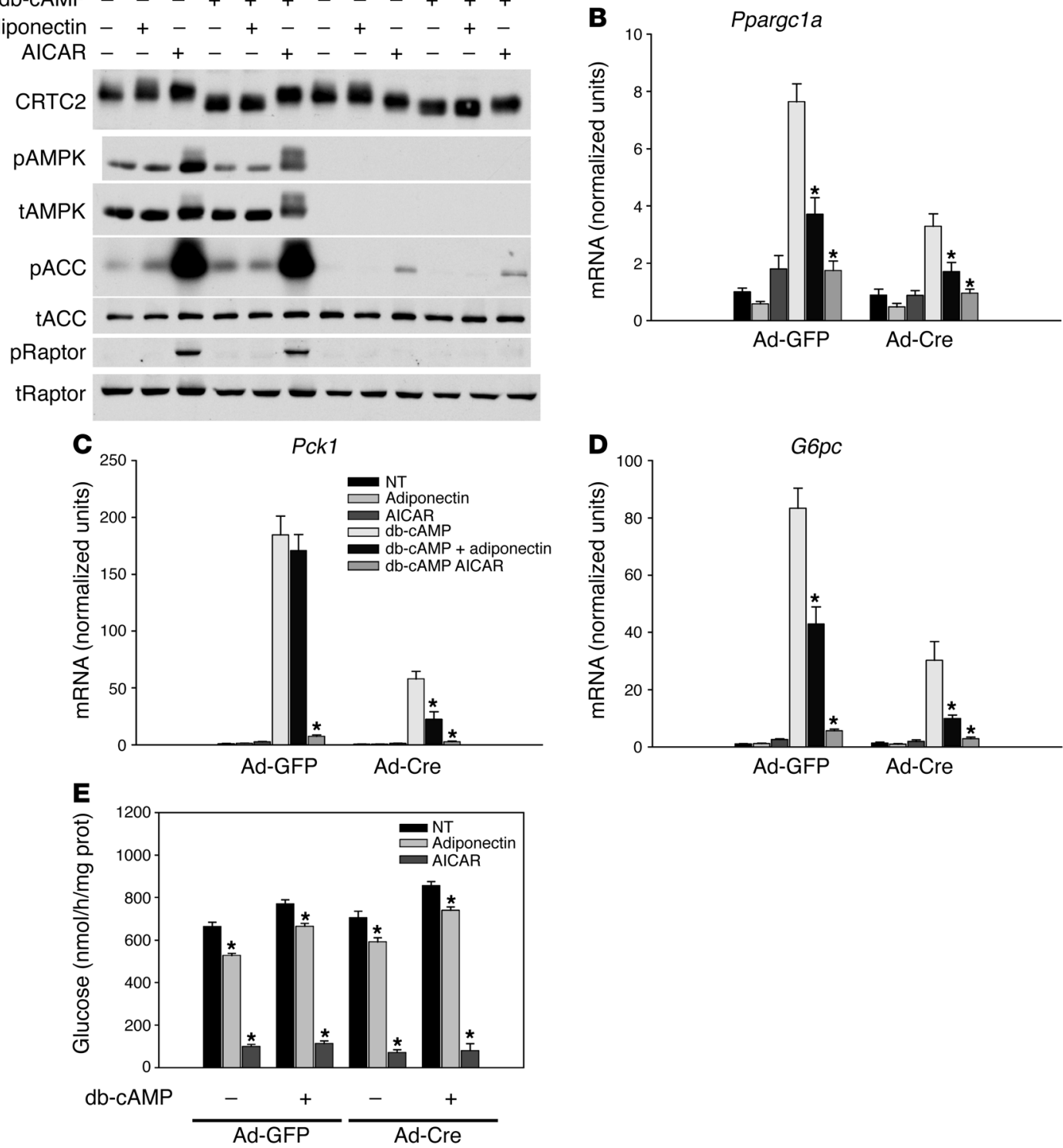

Figure 6

Adiponectin signaling in AMPK $\alpha 1 / \alpha 2-$ deficient primary hepatocytes. (A) Western blots from hepatocytes isolated from AAV-TBG-GFP- or

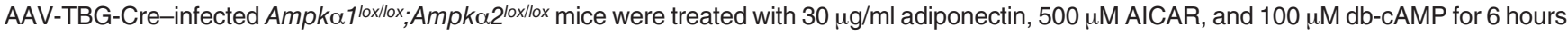
and probed for CRTC2, total and phosphorylated AMPK, total and phosphorylated ACC, and total and phosphorylated Raptor. (B-D) Primary hepatocytes isolated from AAV-TBG-GFP- or AAV-TBG-Cre-infected Ampka1/ox/lox;Ampk $2^{10 x / / 0 x}$ mice were treated with $30 \mu \mathrm{g} / \mathrm{ml}$ adiponectin, $500 \mu \mathrm{M}$ AICAR, and $100 \mu \mathrm{M}$ db-cAMP for 6 hours. Total RNA was isolated and (B) Ppargc1a, (C) Pck1, and (D) G6pc mRNA was quantified and expressed relative to cyclophilin A mRNA and normalized to basal mRNA from GFP-infected hepatocytes. ${ }^{\star} P<0.05$ versus db-cAMP. (E)

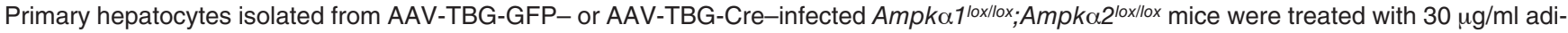
ponectin, $500 \mu \mathrm{M}$ AICAR, and $100 \mu \mathrm{M}$ db-cAMP for 6 hours in glucose-free medium. Glucose production was measured and normalized to total protein. ${ }^{*} P<0.05$ versus untreated with adiponectin or AICAR. All results are expressed as the mean, and error bars represent SEM.

levels for the latter 2 genes (Figure 5, C-E). Importantly, in LKB1-deficient hepatocytes, the effect of adiponectin and AICAR on reducing Ppargc1a and G6pc gene expression was retained. The lack of effect on Pck1 expression was most likely due to the much-reduced response to db-cAMP. In addition to decreasing the mRNA of gluconeogenic genes, adiponectin and AICAR reduced glucose production by hepatocytes isolated from $L K B 1^{\text {lox }} /$ ox mice infected with either Ad-GFP or Ad-Cre (Figure 5F). db-cAMP stimulated glucose production modestly, but again this was decreased by adiponectin or AICAR in both wild-type hepatocytes and those deficient in LKB1 (Figure 5F). These data indicate that LKB1 is not required for cell-autonomous suppression of gluconeogenic gene expression or glucose output by adiponectin in primary hepatocytes.

$A M P K$ is not required for adiponectin-modulated gene expression in primary hepatocytes. To determine whether the LKB1-independent effects of adiponectin on gluconeogenic gene expression were 
nonetheless being mediated by AMPK, we performed studies in primary hepatocytes lacking both the $\alpha 1$ and $\alpha 2$ isoforms of AMPK (Prkaa1 and Prkaa2). Recombination of the floxed AMPK $\alpha 1$ and $\alpha 2$ isoforms by hepatocyte-specific expression of the Cre recombinase led to a complete loss of detectable AMPK $\alpha$ protein and phosphorylation and largely prevented the phosphorylation of the AMPK substrates ACC and Raptor (Figure 6A). In AAV-TBG-GFP-infected Ampk $\alpha 1^{\text {lox } / l o x} ; A m p k \alpha 2^{\text {lox } / l o x}$ hepatocytes, db-cAMP elevated Ppargc1a, Pck1, and G6pc mRNA levels, which were reduced by adiponectin and AICAR. In contrast to LKB1deficient hepatocytes, depletion of AMPK activity by infection of liver with AAV-TBG-Cre did not increase basal gluconeogenic gene expression, supporting the importance of Lkb1 targets other than AMPK in the regulation of hepatic glucose metabolism (Figure 6, B-D). AMPK-deficient hepatocytes still responded to db-cAMP with increased Ppargc1a, Pck1, and G6pc mRNA, and adiponectin and AICAR antagonized this effect. In hepatocytes from $A m p k \alpha 1^{l o x} / l o x ; A m p k \alpha 2^{l o x} / l o x$ mice infected with AAV-GFP, both adiponectin and AICAR decreased both basal and db-cAMP-stimulated glucose production, and this was unchanged in hepatocytes isolated from mice infected with AAV-Cre (Figure 6E).

Loss of CRTC2 fails to prevent adiponectin- and AICAR-induced reductions in CAMP-stimulated gene expression. The CREB coactivator protein CRTC2 is phosphorylated and inhibited by AMPK family kinases and has been proposed as a mediator of LKB1AMPK regulatory input into CREB-dependent transcription (19). Treatment of hepatocytes with db-cAMP increased the electrophoretic mobility of CRCT2, presumably due to cAMPdependent dephosphorylation, and this effect was partially reversed by AICAR but not adiponectin (Figure 5B, Figure 6A, and Supplemental Figure 4A). Removal of hepatic LKB1 converted the CRTC2 protein to the higher mobility, unphosphorylated state even in the absence of db-cAMP, and this was not changed by AICAR or adiponectin, consistent with recent studies examining CRTC2 in LKB1-deficient hepatocytes (Figure 5B and refs. 19, 29, 32). However, interconversion of the 2 forms of CRCT2 by db-cAMP or AICAR was not required for regulation of at least some CREB target genes (Figure 5C). In contrast to LKB1, loss of AMPK had no effect on the basal mobility of CRTC2 or its response to db-cAMP, but led to impairment in the AICAR-induced reversal of increased mobility (Figure 6A). Nonetheless, AICAR regulated CREB target genes in the absence of AMPK (Figure 6, B-D).

The studies described above showed that CRTC2 mobility did not correlate with adiponectin and AICAR modulation of gluconeogenic gene expression; to determine genetically the dependence of the inhibitory actions of AICAR and adiponectin on CRTC2, we measured gluconeogenic gene expression in primary hepatocytes isolated from CRTC2-null mice (33). Loss of CRTC2 protein had no effect on phosphorylation of AMPK or ACC in response to glucagon, AICAR, or adiponectin (Supplemental Figure 4A). As reported previously, CRCT2-deficient hepatocytes were markedly impaired in glucagon-dependent expression of gluconeogenic genes, consistent with the importance of this coactivator to their transcription (33). Nonetheless, the regulation of some of these genes by AICAR and adiponectin was preserved (Supplemental Figure 4, B-D). These results suggest that CRTC2 is dispensable for AICAR- and adiponectin-induced suppression of cAMP-stimulated gene expression.

\section{Discussion}

Adiponectin has been implicated as a major protective factor against the adverse metabolic and cardiovascular consequences of obesity, though its role in normal physiology is still being elucidated $(3,4)$. In this study, we have evaluated the role of the LKB1/AMPK/CRTC2 signaling pathway in the hepatic actions of adiponectin, utilizing a loss-of-function approach for studies both in vivo and in vitro. Consistent with previous work, we found LKB1 relevant to the effects of adiponectin in the liver, but also uncovered a complex interplay between both LKB1-dependent and -independent pathways that control the effects of the hormone on hepatic glucose output. Deletion of hepatic LKB1 by viral expression of Cre recombinase elicited an increase in serum glucose, gluconeogenic gene expression, and hepatic glucose production, and impaired the adiponectin-dependent decrease in serum glucose, largely due to a diminished capacity of adiponectin to lower hepatic glucose production. Surprisingly, this was not due to impairment in adiponectin-induced reductions in gluconeogenic gene expression; while loss of hepatic LKB1 resulted in elevated basal gene expression, the reduction following adiponectin treatment was largely preserved. The deletion of LKB1, AMPK $\alpha$ proteins, and CRTC2, a signaling pathway in the hepatocytelinking energy charge to gluconeogenic gene expression, failed to prevent the reduction of glucose output or gluconeogenic gene expression by adiponectin. Taken together, these data suggest that adiponectin can function through multiple pathways to reduce hepatic glucose output: an LKB1-dependent pathway and an LKB1-, AMPK-, and CRTC2-independent pathway that may involve cAMP-dependent changes in the gene expression of key gluconeogenic genes such as Pck1 and GPc.

Recent studies have suggested that the adiponectin receptors, AdipoR1 and AdipoR2, contain intrinsic ligand-stimulated ceramidase activity independent of LKB1 (34). Elevated hepatic ceramide is associated with many of the phenotypes characteristic of insulin-resistant states, including increased hepatic glucose output and elevated triglyceride levels $(35,36)$. Ceramide directly antagonizes insulin action, largely by preventing phosphorylation and activation of Akt (34). Thus, adiponectin-dependent reductions in ceramide could have an insulin-sensitizing effect, accounting for its action to reduce hepatic glucose output independent of LKB1/ AMPK/CRTC2 signaling $(12,37)$. However, analysis of gene expression following adiponectin treatment in LKB1-null livers revealed a pattern inconsistent with simple insulin sensitization, as not all changes in mRNA levels behaved as expected for enhanced insulin signaling. For example, adiponectin induced a decrease in Srebf1c and increase in Igfbp1 mRNA levels (Figure 4), precisely the opposite of how insulin regulates these genes (38). Thus, it is likely that adiponectin signals through a distinct specialized pathway rather than exclusively enhancing the actions of insulin. Other models of AMPK-independent adiponectin signaling postulate coupling of AdipoRs to the adaptor protein APPL1 $(31,39)$ or activation of P38 MAPK and PPAR $\alpha(40-43)$. It is unclear, however, whether these mechanisms are functional in liver.

As shown here and previously, adiponectin increases intracellular AMP levels, which, based on its effects on the key points of glycolytic and gluconeogenic flux, could account for the LKB1-independent reduction in glucose production (44). Indeed, in a recent study, exposure of LKB1- and AMPK-null hepatocytes to a varied array of pharmacological activators of AMPK implicated the AMP/ ATP ratio but not AMPK activation as critical to the effects of these 
compounds on both hepatic glucose output and gluconeogenic gene expression (29). Importantly, in contrast to the major alterations in AMP/ATP ratio produced by the drugs reported in Foretz et al. (29), our studies revealed only a transient alteration of cellular nucleotide levels following adiponectin treatment (Supplemental Figure 3, B-D); it is therefore unlikely that adiponectin-dependent changes in glucose output and gene expression can be attributed to alterations in adenosine nucleotide levels, which Foretz et al. (29) suggested mediate the AMPK-independent actions of biguanides such as metformin.

While our data reveal that loss of hepatic LKB1 reduces the efficacy of adiponectin to lower blood glucose levels and hepatic glucose production, we are unable to determine whether this is due to an LKB1-dependent, cell autonomous signaling defect or is secondary to the metabolic abnormalities induced by deletion of hepatic LKB1 (45). Adiponectin delivered directly into the central nervous system increased energy expenditure and peripheral fatty acid oxidation in one study and led to increased food intake in another $(9,10)$. It is possible that some of the effects of adiponectin in liver are also mediated by central actions of adiponectin and therefore independent of the liver LKB1/AMPK/CRTC2 pathway. In the current study, the fact that in the intact mouse and isolated primary hepatocytes, adiponectin regulated gene expression in the absence of the LKB1/AMPK/CRTC2 axis suggests strongly the presence of a cell-autonomous mechanism for adiponectin's regulation of gluconeogenic gene expression independent of this pathway.

A potential trivial explanation for LKB1-independent effects in vivo is incomplete excision of LKB1, or proliferation of LKB1-positive, uninfected hepatocytes retaining an intact $L K B 1^{\text {lox }}$ allele. We consider this explanation unlikely for a number of reasons. The greater than 16-day interval between viral infection and experiments provided sufficient time for turnover of LKB1 protein and mRNA, as corroborated by the substantial reduction in LKB1 protein and phosphorylated AMPK as assessed by immunoblot in livers and primary hepatocytes. More importantly, even though much or all of adiponectin's action in vivo was preserved after infection with Ad-Cre, nonetheless there was a significant increase in basal glucose production, emphasizing the efficacy of the deletion. In addition, in hepatocytes isolated from $L K B 1^{l o x} / l o x$ mice injected with Ad-Cre, adiponectin was completely blocked in its ability to stimulate phosphorylation of AMPK. Given the dramatic decrease in LKB1 protein and the binary nature of allelic recombination, any residual LKB1 is likely to represent a small number of uninfected $L K B 1^{l o x} /$ lox hepatocytes rather than low-level expression in all cells and is therefore unlikely to account for the persistent adiponectin-dependent glucose lowering, reduced hepatic glucose output, and regulation of gene expression in vivo and in vitro in LKB1-deficient hepatocytes.

Our studies confirm previous reports that the hepatic loss of LKB1 produces a reduction in basal AMPK phosphorylation with elevated serum glucose levels and impaired glucose tolerance (26). Surprisingly, this elevation in blood glucose was not caused by hepatic insulin resistance as determined by the continued ability of insulin to regulate Akt and Foxo phosphorylation as well as insulin-responsive Srebp1c target genes (Figure 1, A-E, and Figure 4). This result indicates that LKB1 activity suppresses hepatic glucose output utilizing a pathway either parallel to or downstream of Akt/Foxo. Mice lacking hepatic LKB1, like those expressing liverspecific dominant negative AMPK, have elevated blood glucose levels (Figure 1A and refs. 27, 46); interestingly, it has recently been shown that the hepatic-specific deletion of AMPK $\alpha$ subunits does not recapitulate the hyperglycemia phenotype, raising the possibility that dominant negative AMPK can inhibit LKB1 targets other than $\operatorname{AMPK}(27,29)$. Thus, LKB1-dependent kinases other than AMPK, such as SIK2, almost certainly contribute to the maintenance of basal glycemia. In contrast, it is unlikely that these kinases account for hepatic responsiveness to adiponectin or AICAR in the absence of AMPK, as deletion of AMPK also leads to loss of phosphorylation of the canonical AMPK substrates Raptor and ACC (Figure 6A). Surprisingly, whereas deletion of hepatic Lkb1 leads to increased hepatic glucose output, it actually antagonizes the ability of db-cAMP to activate Pck1 gene expression (Figure 5D). It is unclear whether this reflects a requirement for a Lkb1-dependent pathway in glucagon signaling or represents a novel feedback response to prolonged elevated glucose production.

We have presented 2 pieces of evidence that suggest that the transcriptional regulator CRTC2 is not a primary input for both AICAR and adiponectin repression of PKA-stimulated gene expression. First and most importantly, hepatocytes from CRTC2-null mice demonstrated a robust AICAR- and adiponectin-induced repression of the principal PKA target gene, Ppargc1a (Supplemental Figure 4B). Second, both LKB1- and AMPK $\alpha$-null hepatocytes exhibited a lack of correlation between CRTC2 electrophoretic mobility and adiponectin- and AICAR-mediated repression of db-cAMP-stimulated gluconeogenic mRNA expression (Figure 5C and Figure 6B). Consistent with the role of CRTC2 as functioning downstream of LKB1 to coactivate CREB activity, LKB1-null cells exhibited dephosphorylated CRTC2 and high basal gluconeogenic gene expression (Figure 5, B and C), and CRTC2-null cells had reduced glucagon-stimulated gene expression (Supplemental Figure 4B), as reported previously (33). Whereas other studies have implicated AMPK or SIK2 phosphorylation of CRTC2 as an important mechanism for repression of the CREB-signaling pathway, our results suggest that other unknown LKB1-, AMPK-, and CRTC2independent mechanisms also contribute to gluconeogenic gene expression by adiponectin and other canonical AMPK activators $(19,45)$. The effect of these activators is likely to be downstream of PKA signaling, as both adiponectin and AICAR failed to suppress both glucagon- and db-cAMP-induced PKA phosphorylation of target proteins (Supplemental Figure 5).

In summary, our data support a role for LKB1 in some of the hepatic actions of adiponectin, showing that the AMPKK LKB1 is required for adiponectin's full glucose-lowering effect. These studies, however, also indicate an LKB1-independent pathway that persists in LKB1-deficient livers, in which adiponectin regulates the suppression of glucose output and gluconeogenic gene expression. As the latter effect of adiponectin does not require LKB1, AMPK, or CRTC2 in primary hepatocytes, we propose a cell-autonomous pathway by which adiponectin controls both gluconeogenic gene expression and hepatic glucose output independently of these signaling molecules.

\section{Methods}

Mice. LKB1 $1^{l o x} /$ lox mice were provided by R.A. DePinho (Harvard Medical School, Boston, Massachusetts, USA) (30). Mice were housed in a facility on a 12-hour light/12-hour dark cycle with free access to food and water. All procedures were reviewed and approved by the Institutional Animal Care and Use Committee at the University of Pennsylvania. The derivation of the AMPK $\alpha$ and CRTC2 (TORC2) mice has been described previously $(33,47)$. Mice were genotyped as described. Blood glucose values were mea- 
sured with OneTouch Ultra glucose analyzer. Serum insulin was measure using the QuantiChrom ultrasensitive insulin ELISA. Hepatic triglycerides were measured using the Infinity triglyceride reagent (Thermo). Proteins were quantified by BCA assay (Pierce).

Viruses and infections. Adenoviruses expressing Cre recombinase and GFP (Ad-Cre and Ad-GFP) and Adeno-associated viruses expressing Cre recombinase or GFP from a TBG promoter (AAV-TBG-Cre and AAV-TBG-GFP) were amplified in the University of Pennsylvania Gene Therapy Vector Core. Mice were infected with the indicated viruses by retroorbital injection, and studies were performed 16-22 days after infection.

Production of adiponectin. Adiponectin was produced as previously reported using 293 cells (12) and was primarily present in HMW (High Molecular Weight) form, with some hexameric and trimeric protein also present (Supplemental Figure 6). Adiponectin preparations were assessed for activity through glucose-lowering effects in wild-type mice. Cell culture adiponectin activity was confirmed by immunodepletion of AMPK-activating efficacy from adiponectin protein preparations using anti-adiponectin antibodies.

Acute in vivo effects of adiponectin. Mice were fasted for 1 hour ( $9 \mathrm{am}-10 \mathrm{am})$, tail blood glucose was measured using a glucometer (OneTouch Ultra), adiponectin $34 \mu \mathrm{g} / \mathrm{g}$ body weight or vehicle was injected i.p., and tail-blood glucose was monitored at the indicated times. For Western blotting and RNA studies, mice were fasted for 1 hour ( $9 \mathrm{am}-10 \mathrm{am}$ ), and adiponectin $(34 \mu \mathrm{g} / \mathrm{g})$ or vehicle was injected i.p.; mice were then sacrificed by cervical dislocation and their livers were rapidly freeze clamped and processed for subsequent studies.

Hyperinsulinemic englycemic clamp. Clamp experiments were performed in the Diabetes and Endocrinology Research Center (DERC) Mouse Phenotyping, Physiology and Metabolism Core as previously reported (48).

Gene expression analysis. For tissues, mice were sacrificed following the indicated treatments and tissues were rapidly frozen in precooled metal clamps and frozen in liquid nitrogen. RNA was extracted from $100 \mathrm{mg}$ of tissue with Trizol (Invitrogen) in a TissueLyser, and RNA reverse transcribed to cDNA using a RetroScript kit (Ambion). The following qPCR primers were used: qPCR primers: G6Pase, ATGAACATTCTCCATGACTTTGGG and GACAGGGAACTGCTTTATTATAGG; PEPCK, GTGGGAGTGACACCTCACAGC and AGGACAGGGCTGGCCGGGACG; $P G C 1 \alpha$, GAGAATGAGGCAAACTTGCTAGCG and TGCATGGTTCTGAGTGCTAAGACC; cyclophilin A, TGGAGAGCACCAAGACAGACA and TGCCGGAGTCGACATGAT. For global gene analysis, a custom TaqMan low-density array was used to quantify a panel of metabolically important genes in liver on an ABI instrument. For primary hepatocytes, cells were plated overnight in M199 medium containing 0.05\% BSA, $100 \mathrm{nM}$ dexamethasone, $100 \mathrm{nM} \mathrm{T} 3$, and $1 \mathrm{nM}$ insulin. After 36 hours, the medium was refreshed and the indicated treatments were delivered. Cells were lysed 6 hours after treatment, and RNA was extracted then reverse transcribed to cDNA using a RetroScript kit (Ambion). cDNA was analyzed by quantitative PCR using the indicated primers.

Primary hepatocytes. Primary hepatocytes were isolated from mice using a modified 2-step perfusion method (49) using Liver Perfusion Media and Liver Digest Buffer (Invitrogen). Cells were plated in collagen I-coated 6- or 12-well plated (at 2 or 1 million cells per well, respectively) in M199 medium plus 10\% FBS plus penicillin/streptomycin. After 3 hours of attachment, the medium was replaced with the appropriate assay medium.

Quantification of adenine nucleotides in primary hepatocytes. Primary hepatocytes isolated as described above were plated on 6-cm collagen-coated Petri dishes and 24 hours after isolation were treated with compounds as indicated. Cells were scraped from the plate into ice-cold PBS, rapidly pelleted with lowspeed centrifugation, and lysed with $0.5 \mathrm{M}$ perchloric acid. The soluble fraction was neutralized with 0.25 volumes $2 \mathrm{M} \mathrm{KOH}, 1 \mathrm{M} \mathrm{PO}_{4}, \mathrm{pH} 7.8$, filtered with syringe-tip $0.45-\mu \mathrm{m}$ filters (Millipore), and frozen in liquid nitrogen. Total protein was measured from perchloric acid-precipitated materials by solubilization in $6 \mathrm{M}$ urea followed by BCA assay. Perchloric acid-soluble materials were separated by isocratic elution in an ion-pairing reverse-phase HPLC system (buffer used was $200 \mathrm{mM} \mathrm{KH}_{2} \mathrm{PO}_{4}$, pH 6.25, 5 mM tetrabutylammonium phosphate [TBAP], $3 \%$ acetonitrile). AMP, ADP, and ATP peak areas were calculated and converted to molar amounts through comparison with standard curves generated from quantification of known quantities of pure nucleotides separated under identical conditions.

Glucose output. Hepatocytes isolated from mice fasted overnight were plated onto collagen-coated 12 -well plates at a density of $5 \times 10^{5}$ cells/well in serum-free M199 medium. After cells had attached (2 hours), the medium was changed to glucose and phenol red-free DMEM with the addition of $10 \mathrm{mM}$ lactate, $1 \mathrm{mM}$ pyruvate, $5 \mathrm{mM}$ glutamine, and compounds as indicated. Cells were incubated for 6 hours and medium collected. Total protein was extracted with $1 \%$ Triton X-100 and quantified by BCA assay (Pierce). Medium glucose was assayed using a hexokinase-based glucose assay (Sigma-Aldrich).

Statistics. All 2-group comparisons were deemed statistically significant by unpaired 2-tailed Student's $t$ test if $P<0.05$. All in vitro studies are either compilation of 3 independent experiments or representative of 3 independent experiments. Number of mice used in each in vivo condition is indicated in the figure legends.

\section{Acknowledgments}

We would like to thank R.A. DePinho for providing the $L K B 1^{l o x} /$ lox mice. This work was supported by NIH grants R01 DK56886 and P01 DK49210 (to M.J. Birnbaum), P01 DK049210 (to K.H. Kaestner), and F32 DK079572 (to R.A. Miller) and the Association pour l'Etude des Diabètes et des Maladies Métaboliques (ALFEDIAM) (to M. Foretz), the Programme National de Recherche sur le Diabète (PNRD) (to M. Foretz and B. Viollet), and the Institut Benjamin Delessert (to M. Foretz). The Transgenic/Knockout, Mouse Phenotyping, and Biomarker Cores of the University of Pennsylvania Diabetes and Endocrinology Research Center (NIH grant P30 DK19525) were instrumental in this work.

Received for publication November 29, 2010, and accepted in revised form April 11, 2011.

Address correspondence to: Morris J. Birnbaum, Clinical Research Building, Rm 310, 415 Curie Blvd., Philadelphia, Pennsylvania 19104, USA. Phone: 215.898.5001; Fax: 215.573.9138; E-mail: birnbaum@mail.med.upenn.edu.
1. Hu E, Liang P, Spiegelman BM. AdipoQ is a novel adipose-specific gene dysregulated in obesity. J Biol Chem. 1996;271(18):10697-10703.

2. Scherer PE, Williams S, Fogliano M, Baldini G, Lodish HF. A novel serum protein similar to C1q, produced exclusively in adipocytes. $\mathrm{J}$ Biol Chem. 1995;270(45):26746-26749.

3. Kumada M, et al. Association of hypoadiponectinemia with coronary artery disease in men. Arte- rioscler Thromb Vasc Biol. 2003;23(1):85-89.

4. Hotta K, et al. Plasma concentrations of a novel, adipose-specific protein, adiponectin, in type 2 diabetic patients. Arterioscler Thromb Vasc Biol. 2000;20(6):1595-1599.

5 . Kubota N, et al. Adiponectin stimulates AMPactivated protein kinase in the hypothalamus and increases food intake. Cell Metab. 2007;6(1):55-68. 6. Ohashi K, et al. Adiponectin I164T mutation is associated with the metabolic syndrome and coronary artery disease. J Am Coll Cardiol. 2004; 43(7):1195-1200.

7. Yamauchi $\mathrm{T}$, et al. The fat-derived hormone adiponectin reverses insulin resistance associated with both lipoatrophy and obesity. Nat Med. 2001;7(8):941-946.

8. Qi Y, et al. Adiponectin acts in the brain to decrease body weight. Nat Med. 2004;10(5):524-529. 
9. Fruebis J, et al. Proteolytic cleavage product of $30-\mathrm{kDa}$ adipocyte complement-related protein increases fatty acid oxidation in muscle and causes weight loss in mice. Proc Natl Acad Sci U S A. 2001;98(4):2005-2010.

10. Combs TP, Berg AH, Obici S, Scherer PE, Rossetti L. Endogenous glucose production is inhibited by the adipose-derived protein Acrp30. J Clin Invest. 2001;108(12):1875-1881.

11. Ceddia RB, Somwar R, Maida A, Fang X, Bikopoulos G, Sweeney G. Globular adiponectin increases GLUT4 translocation and glucose uptake but reduces glycogen synthesis in rat skeletal muscle cells. Diabetologia. 2005;48(1):132-139.

12. Berg AH, Combs TP, Du X, Brownlee M, Scherer PE. The adipocyte-secreted protein Acrp30 enhances hepatic insulin action. Nat Med. 2001;7(8):947-953.

13. Xu A, Wang Y, Keshaw H, Xu LY, Lam KS, Cooper GJ. The fat-derived hormone adiponectin alleviates alcoholic and nonalcoholic fatty liver diseases in mice. J Clin Invest. 2003;112(1):91-100.

14. Nawrocki AR, et al. Mice lacking adiponectin show decreased hepatic insulin sensitivity and reduced responsiveness to peroxisome proliferator-activated receptor gamma agonists. J Biol Chem. 2006; 281(5):2654-2660

15. Kim JY, et al. Obesity-associated improvements in metabolic profile through expansion of adipose tissue. J Clin Invest. 2007;117(9):2621-2637.

16. Viollet B, et al. AMP-activated protein kinase in the regulation of hepatic energy metabolism: from physiology to therapeutic perspectives. Acta Physiol (Oxf). 2009;196(1):81-98.

17. Hardie DG, Hawley SA. AMP-activated protein kinase: the energy charge hypothesis revisited. Bioessays. 2001;23(12):1112-1119.

18. Carling D, Clarke PR, Zammit VA, Hardie DG. Purification and characterization of the AMP-activated protein kinase. Copurification of acetyl-CoA carboxylase kinase and 3-hydroxy-3-methylglutarylCoA reductase kinase activities. Eur J Biochem. 1989; 186(1-2):129-136

19. Koo SH, et al. The CREB coactivator TORC2 is a key regulator of fasting glucose metabolism. Nature. 2005;437(7062):1109-1111.

20. Woods A, et al. LKB1 is the upstream kinase in the AMP-activated protein kinase cascade. Curr Biol. 2003;13(22):2004-2008.

21. Shaw RJ, et al. The tumor suppressor LKB1 kinase directly activates AMP-activated kinase and regulates apoptosis in response to energy stress. Proc Natl Acad Sci U S A. 2004;101(10):3329-3335.

22. Hurley RL, Anderson KA, Franzone JM, Kemp BE, Means AR, Witters LA. The Ca2+/calmodu- lin-dependent protein kinase kinases are AMPactivated protein kinase kinases. J Biol Chem. 2005;280(32):29060-29066.

23. Hawley SA, et al. Complexes between the LKB1 tumor suppressor, STRAD alpha/beta and MO25 alpha/beta are upstream kinases in the AMP-activated protein kinase cascade. J Biol. 2003;2(4):28.

24. Koh HJ, et al. Skeletal muscle-selective knockout of LKB1 increases insulin sensitivity, improves glucose homeostasis, and decreases TRB3. Mol Cell Biol. 2006;26(22):8217-8227.

25. Sakamoto K, et al. Deficiency of LKB1 in skeletal muscle prevents AMPK activation and glucose uptake during contraction. EMBO J. 2005;24(10):1810-1820.

26. Shaw RJ, et al. The kinase LKB1 mediates glucose homeostasis in liver and therapeutic effects of metformin. Science. 2005;310(5754):1642-1646.

27. Yamauchi T, et al. Adiponectin stimulates glucose utilization and fatty-acid oxidation by activating AMP-activated protein kinase. Nat Med. 2002; 8(11):1288-1295

28. Awazawa M, et al. Adiponectin suppresses hepatic SREBP1c expression in an AdipoR1/LKB1/AMPK dependent pathway. Biochem Biophys Res Commun. 2009;382(1):51-56

29. Foretz M, et al. Metformin inhibits hepatic gluconeogenesis in mice independently of the LKB1/ AMPK pathway via a decrease in hepatic energy state. J Clin Invest. 2010;120(7):2355-2369.

30. Bardeesy N, et al. Loss of the Lkb1 tumour suppressor provokes intestinal polyposis but resistance to transformation. Nature. 2002;419(6903):162-167.

31. Zhou L, et al. Adiponectin activates AMP-activated protein kinase in muscle cells via APPL1/ LKB1-dependent and phospholipase C/Ca2+ $\mathrm{Ca} 2+/$ calmodulin-dependent protein kinase kinase-dependent pathways. J Biol Chem. 2009; 284(33):22426-22435.

32. Hashimoto YK, Satoh T, Okamoto M, Takemori H. Importance of autophosphorylation at Ser186 in the A-loop of salt inducible kinase 1 for its sustained kinase activity. J Cell Biochem. 2008; 104(5):1724-1739.

33. Le Lay J, Tuteja G, White P, Dhir R, Ahima R, Kaestner KH. CRTC2 (TORC2) contributes to the transcriptional response to fasting in the liver but is not required for the maintenance of glucose homeostasis. Cell Metab. 2009;10(1):55-62.

34. Holland WL, et al. Receptor-mediated activation of ceramidase activity initiates the pleiotropic actions of adiponectin. Nat Med. 2010;17(1):55-63.

35. Yang G, Badeanlou L, Bielawski J, Roberts AJ, Hannun YA, Samad F. Central role of ceramide biosyn- thesis in body weight regulation, energy metabolism, and the metabolic syndrome. Am J Physiol Endocrinol Metab. 2009;297(1):E211-E224.

36. Yew NS, et al. Increased hepatic insulin action in dietinduced obese mice following inhibition of glucosylceramide synthase. PLoS One. 2010;5(6):e11239.

37. Wang Y, Xu A, Knight C, Xu LY, Cooper GJ. Hydroxylation and glycosylation of the four conserved lysine residues in the collagenous domain of adiponectin. Potential role in the modulation of its insulin-sensitizing activity. J Biol Chem. 2002; 277(22):19521-19529.

38. Gross DN, van den HeuvelAPJ, Birnbaum MJ. The role of FoxO in the regulation of metabolism. Oncogene. 2008;27(16):2320-2336.

39. Mao X, et al. APPL1 binds to adiponectin receptors and mediates adiponectin signalling and function. Nat Cell Biol. 2006;8(5):516-523.

40. Yoon MJ, Lee GY, Chung JJ, Ahn YH, Hong SH, Kim JB. Adiponectin increases fatty acid oxidation in skeletal muscle cells by sequential activation of AMPactivated protein kinase, $\mathrm{p} 38$ mitogen-activated protein kinase, and peroxisome proliferator-activated receptor alpha. Diabetes. 2006;55(9):2562-2570.

41. Yamauchi T, et al. Targeted disruption of AdipoR1 and AdipoR2 causes abrogation of adiponectin binding and metabolic actions. Nat Med. 2007; 13(3):332-339.

42. Yamauchi T, et al. Cloning of adiponectin receptors that mediate antidiabetic metabolic effects. Nature. 2003;423(6941):762-769.

43. Wanninger J, et al. Adiponectin-stimulated CXCL8 release in primary human hepatocytes is regulated by ERK1/ERK2, p38 MAPK, NF-kappaB, and STAT3 signaling pathways. Am J Physiol Gastrointest Liver Physiol. 2009;297(3):G611-G618.

44. Miller RA, Birnbaum MJ. An energetic tale of AMPK-independent effects of metformin. J Clin Invest. 2010;120(7):2267-2270.

45. Liu Y, et al. A fasting inducible switch modulates gluconeogenesis via activator/coactivator exchange. Nature. 2008;456(7219):269-273.

46. Viana AY, et al. Role of hepatic AMPK activation in glucose metabolism and dexamethasone-induced regulation of AMPK expression. Diabetes Res Clin Pract. 2006;73(2):135-142.

47. Viollet B, et al. The AMP-activated protein kinase alpha2 catalytic subunit controls whole-body insulin sensitivity. J Clin Invest. 2003;111(1):91-98.

48. Qi Y, et al. Loss of resistin improves glucose homeostasis in leptin deficiency. Diabetes. 2006; 55(11):3083-3090.

49. Seglen PO. Preparation of isolated rat liver cells. Methods Cell Biol. 1976;13:29-83. 